\title{
Selection versus adaptation: network diversification and the origins of life, ageing and cancer
}

\author{
H.V. Westerhoff \\ Synthetic Systems Biology and Nuclear Organization, University of Amsterdam \\ Molecular Cell Physiology, Vrije Universiteit Amsterdam \\ Systems Biology, The University of Manchester \\ Infrastructure Systems Biology Europe. NL \\ e-mail:h.v.westerhoff@uva.nl
}

Key words: networks, functional heterogeneity, drug resistance, flux balance analysis, stochastic modelling

Networking enables functions that are otherwise thermodynamically impossible, such as the synthesis of ATP, proteins and DNA. We shall here highlight a lesser known function of networking, i.e. diversification. Network diversification followed by selection, sprouted the tree of Life, but that very tree hides a forest of diversity. Early Life on this planet may have benefitted from diversification of the redox network around acetogenesis. Flux Balance Analysis (FBA) of the genome-wide metabolic network of Cl. Ljungdahlii reveals carbon fixation at various ATP/acetate stoicheiometries. This may have enabled early organisms to survive the erratic environmental conditions by shifting gears. The flexible ATP yield enabled by the Warburg effect may help do so for tumor cells.

Our organs are subject to a drizzle of somatic mutations, which leads to cell diversification with age. We shall review an FBA methodology that simulates this and then demonstrate that this may initially enhance the metabolic versatility of organs such as T-cells and liver, in the absence of adaptation. More somatic mutations would cause loss of function and ageing. And, subpopulations of asocial cells would develop into tumors with Warburg and the new WarburQ (i. e. glutamine dependent) phenotypes.

Likewise, noise such as required by the third law of thermodynamics should diversify cell populations. FISH and deep sequencing experiments show even stronger noise than this, which should thereby be subject to regulation, e.g. through transcription bursting. We shall show that even though such noise varies with time, it may be selectable and may lead to drug resistance of tumor cell populations, either because of nonlinearities or because of the 'Waddington' genetic landscape, a remnant of developmental biology. 